\title{
Die Behinderten in Herten
}

\author{
Von Eduard Seidler
}

Die nachfolgenden Überlegungen betreffen eine Gruppe von Menschen, von denen nie so ganz klar war - im Grunde bis heute nicht -, ob sie ein Gegenstand der Medizin sind und wer überhaupt für sie zuständig ist. Das Generalthema dieses Heftes bietet die Möglichkeit, eine der vielen Institutionen vorzustellen, in denen die Gesellschaft versucht hat, diese Art von Mitmenschen aufzufangen; der kleine Ort Herten, am badischen Ufer des Oberrheines bei Rheinfelden gelegen, ist so nahe zur Schweiz gelegen, daß der Blick aus den Fenstern auf Kaiseraugst und das schweizerische Rheinfelden fällt. Außerdem ist die Gründung, die Struktur und die Funktion dieser Behindertenanstalt bis heute so intensiv mit der Schweiz verbunden und von ihr geprägt, daß auch dieser Zusammenhang es wert ist, dargestellt zu werden.

Am Anfang der Gründungsgeschichte steht ein kleines, später immer wieder zitiertes Ereignis, das trotz seines anekdotischen Charakters sehr gut die Gesamtproblematik beleuchtet. Es ist dies der Gang des nachmaligen Anstaltsgründers Karl Rolfus (1819-1907), Ortspfarrers in Herten, mit der Generaloberin der Barmherzigen Schwestern vom Heiligen Kreuz aus Ingenbohl (Kanton Schwyz), Maria Theresia Scherer, in den Fastnachtstagen 1878 vom Bahnhof zum Pfarramt in Herten. In seiner Chronik notiert Rolfus sehr knapp: ... «beim Begegnen einiger cretinenmäßiger Persönlichkeiten auf dem Wege machte sie ohne jede weitere Veranlassung die Bemerkung: Hier könnte man auch eine Cretinenanstalt errichten.» Rolfus hat unmittelbar reagiert und auch die kleine Szene in den schon wenige Tage später, am Josefstag, dem 19. März 1878, von Rolfus und dem Säckinger Pfarrer Danner entworfenen «Prospect über die Errichtung einer PrivatPfleg-Anstalt für Kretinen in Herthen» aufgenommen. Dort heißt es in ausgeschmückter Form: «Wenn man diese kleinen zwergartigen Gestalten sieht mit großem Kopf, niedriger Stirn, breitem, offenstehenden Mund, dicken Lippen und vorstehendem Oberkiefer, mit ihren glotzenden Augen und lallender Zunge, wie sie den Vorübergehenden mit blödsinnigem Lächeln oft angrinzen und sich mühsam weiterschleppen, so wird man von tiefstem Mitleid ergriffen.» Noch der erste Jubiläumsjahresbericht von 1904 zur 25-Jahr-Feier beginnt mit der gleichen Episode und bringt dann einen 
Text, in dem alle jene Beschreibungen vorkommen, die uns als Gemisch wissenschaftlicher und umgangssprachlicher Termini immer wieder entgegentreten, wenn derlei Behinderte beschrieben werden. Die Cretinen sind «stumpfsinnig», "geistig niedergedrückt», sie sind «armselig» und «auf niedriger Stufe», zeigen bei «abstoßender körperlicher Deformität» vielleicht «noch einige Bildungsfähigkeit», sind aber alle «gewöhnlich sich und den Ihrigen, ihrer Umgebung und den Gemeinden zur Last». Man müsse sie daher vor allem vor der «Verwahrlosung oder Verthierung» bewahren, mahnt im Juli 1878 das an der Gründung beteiligte Schweizer Mutterhaus der Schwestern vom Heiligen Kreuz und deutet damit an, wie schwierig es zu sein scheint, diese «Unglücklichen» überhaupt zu den Menschen zu zählen.

Wenn wir uns vergegenwärtigen, daß Kretinismus zur Zeit der Anstaltsgründung zwar nicht mehr wissenschaftlich, aber umgangssprachlich immer noch als Oberbegriff für angeborene oder in der Kindheit erworbene geistige und körperliche Schäden galt, daß der «Kretin» noch als Synonym für den «Idioten» stand, dann wird klar, daß es sich um jene Varianten der menschlichen Gesellschaft handelte, die von Kind auf in nicht mehr verstehbarer Weise anders waren als die anderen. Wenn es stimmt, daß «Cretin» von «christianus» abgeleitet ist und die vollzogene Taufe kennzeichnen soll, dann scheint diese auch zunächst als das primär anzustrebende Ziel im Vordergrund des Interesses gestanden zu haben. Es waren jene Kinder, die in die gängigen sozialen Institutionen wie Familie, Schule, Krankenhäuser und Pflegeheime für Gebrechliche und Alte nicht paßten und für die man - um noch einmal Rolfus zu zitieren - andere Mittel und Wege finden mußte, «um ihnen ein menschenwürdiges Dasein zu verschaffen». Für den Anstaltsgründer hieß dies, «derlei Unglückliche für die menschliche Gesellschaft noch möglichst nützlich zu machen, sie vor Ausschreitungen zu bewahren und sie soweit als möglich zur Erfüllung der gewöhnlichsten Pflichten eines Christen anzuhalten».

Das Phänomen der Behinderung ist auch im Zeitalter der Massenmedien noch nicht zur zentralen Gesellschaftsaufgabe geworden; von den über 4 Millionen Behinderten in der BRD sind immer noch nur diejenigen sichtbar, von denen Rolfus sagen würde, daß es «Kretinen auf höherer Stufe» seien. Für die anderen hat sich aus ganz bestimmten Gründen eine Typologie bestimmter Zufluchts- und Pflegeinstitutionen gebildet, die auch baulich am Rande der Gesellschaft angesiedelt und damit dem Blick entzogen sind.

Warum ist dies so? Warum befürchtet 1878 der Gemeinderat von Herten, durch die «Aufnahme fremder krüppelhafter Personen» seien z. B. junge 
Frauen und die zukünftige Generation Gefahren ausgesetzt»? W arum erregt sich 1980 eine Gemeinde und bildet eine Bürgerinitiative, um gegen die geplante Errichtung eines Behindertenzentrums zu protestieren? Was haben wir, die Anderen, in unserer Vorstellungswelt und unserem Empfinden für Mechanismen entwickelt bzw. bewahrt, die die meisten von uns daran hindern, emotionsfrei, besser: angstfrei mit dem Problem der schweren Behinderung umzugehen? Was ist in der Vergangenheit geschehen und vor allem, was ist nicht geschehen?

Dies sind wissenschaftliche und praktische Herausforderungen an den Medizinhistoriker - nicht im Sinne historischer Annotationen, sondern im Sinne eines konstruktiven Beitrages zur aktuellen Behindertenproblematik. Es geht um die Gründe für das beharrliche Fortbestehen jener wenigen, aber elementaren Fragen, die bis heute die Geschichte der Behindertenfürsorge durchziehen und die wie folgt lauten: Handelt es sich beim Umgang mit dem Behinderten um ein Problem von Heilbarkeit und Unheilbarkeit, von Pflege und Bewahrung oder von Erziehung und Bildung; handelt es sich - vor allem anderen - um ein Herausgefordertsein durch Störung und Hilflosigkeit oder um eine Herausforderung zur Akzeptation mitmenschlichen Unglücks?

Das Problem von Heilbarkeit und Unheilbarkeit steht am Anfang der neuzeitlichen Beschäftigung mit den Behinderten und markiert seit etwa 1850 den Standort der medizinischen und öffentlichen Betrachtung bis in unsere Tage. Wir wissen, daß alle Bemühungen und Reformen der Neuzeit nur dann richtig verstanden werden können, wenn man die Einstellungen von Medizin und Gesellschaft zu den damals sog. Idioten einerseits und den sog. Geisteskranken andererseits vergleicht. Wir finden gerade in diesem Bereich einen Höhepunkt der Diskussion in jenem Zeitabschnitt, welcher der Gründung von Herten und anderer Institutionen unmittelbar vorangeht und die Zeitsignatur prägt. Mit der zunehmenden Erforschung der psychischen Störungen und ihrer Fixierung auf die Vorstellung von einer Gehirnkrankheit um die Mitte des 19. Jahrhunderts ging eine Anerkennung des damals sogenannten Irren als geisteskrank einher. Damit verband sich auch im öffentlichen Bewußtsein die Hoffnung auf eine Heilungsmöglichkeit, und dem Irren wurde wie allen Kranken ein Recht auf therapeutische Bemühung und soziale Anteilnahme zugebilligt.

Von diesen Bemühungen blieben die nach wie vor so genannten «Idioten» ausgespart. In der Allgemeinen Zeitschrift für Psychiatrie schrieb der Psychiater Heinrich Damerow 1858 einen Grundsatzartikel «zur Cretinenund Idiotenfrage» und nahm eine Einteilung vor, deren nicht nur wissen- 
schaftliche, sondern langwirkende soziale Relevanz nicht deutlich genug aufgezeigt werden kann. Er wies darauf hin, daß es sich bei den Insassen der neugegründeten Heil- und Pflegeanstalten um «Seelenkranke», d.h. um früher gesunde Menschen handle; dagegen hätten «die echten Cretinen und Idioten keine natürliche menschliche Entwicklung, keine persönliche Lebensgeschichte». «Körperlich ... verkümmert, mißgeboren, mißgebildet sind und bleiben sie Mißgeschöpfe, selbst im thierischen Leben, Mißmenschen, bei denen das menschliche psychische und geistige Leben keine immanente Stätte hat.» Und Damerow fügt die Frage hinzu - wohlgemerkt vor den Zeiten Darwins und des Sozialdarwinismus-, «ob die zur Einrichtung und Unterhaltung von Cretinen- und Idiotenanstalten erforderlichen außerordentlichen Mittel und Kräfte in einem nur einigermaßen berechtigenden Verhältnis stehen zu den Zwecken an sich, zu den Verheißungen und Versicherungen der Erfolge in betreff der Erziehung, Bildung und Heilung der Cretinen, Idioten und Blödsinnigen von Kindheit an».

Es gab in der Tat eine ganze Reihe zeitgenössischer Bestrebungen, Behandlungs-, Erziehungs- und Bildungsversuche an geistig Behinderten vorzunehmen. Erinnert sei an die Bemühungen des Pariser Arztes Itard um das sog. Wildkind von Aveyron, an seinen Schüler Edouard Séguin, der in Frankreich und später in den USA eine systematische Schwachsinnigenerziehung entwarf; erinnert sei an Johann Jakob Guggenbühl, der 1841 auf dem Abendberg mit einer vielbeachteten Kretinismusbehandlung begann, und letztlich an Deinhard und Georgens in Wien, die in ihrer kleinen Institution erste Versuche zur Koedukation geistig behinderter und gesunder Kinder begonnen hatten. All dies schien jetzt als in den Anfängen gescheitert angesehen zu werden; «es gibt nichts wiederherzustellen», so noch einmal Damerow, «was nie dagewesen ist». Folglich gehe es nicht um die Errichtung von Heil-, Erziehungs- und Bildungsanstalten, sondern um die Errichtung von sog. Aufbewahrungs-, Bewahr-, Behütungs-, Haltebzw. Pflegeanstalten für Idioten. Dies sei überdies ausschließlich die Aufgabe der Privatwohltätigkeit, ja der Barmherzigkeit von Privaten, Vereinen, Corporationen etc. ...»; «aus Staats- bzw. ständischen Mitteln können die Einrichtungen nicht verlangt werden».

Solche Äußerungen sind keine vergangenen Kuriosa; vielmehr werden hier im Keim Probleme sichtbar, die fortan das Behindertenproblem nicht mehr verlassen sollen: Man wird in der Folgezeit immer wieder diskutieren, ob der Schwachsinn eine Krankheit oder ein Zustand sei; man wird sich streiten, ob der Umgang mit ihnen eine ärztliche, eine pädagogische oder 
eine pflegerische Aufgabe sei; man wird sich um den Sinn von Bildungsfähigkeit streiten, und man wird im Verhandeln um Direktorenposten der Anstalten bis heute nie genau wissen, ob man einem Geistlichen, einem Pädagogen oder einem Arzt den Vorzug geben soll.

Während z.B. Frankreich entsprechende Einrichtungen an die großen Irrenanstalten (Salpêtrière, Bicêtre) oder an die Hospices des Incurables angegliedert hat, waren es in Deutschland die beiden großen Konfessionen, die innerhalb der Sozialbewegung des 19. Jahrhunderts der Behindertenfürsorge einen starken Auftrieb gegeben haben. Seit der berühmten Stegreifrede Johann Heinrich Wicherns im Revolutionsjahr 1848 in Wittenberg entstand in beiden Konfessionen eine blühende Aktivität in Vereinsgründungen für Arme und Kranke, für entlassene Strafgefangene und verwahrloste Kinder, für Lehrlinge und Gesellen; man gründete Sparkassen, Heime, Kinderbewahranstalten und Industrieschulen und eröffnete eine bedeutende Periode von Anstaltsgründungen zu verschiedenen $Z$ wecken.

Auch in der Schweiz geschah Gleichsinniges, und ich erinnere im Hinblick auf Herten an den berühmten sogenannten «Schweizer Schul-, Caritas- und Sozialapostel» Theodosius Florentini, eigentlich Florintöni (geb. 1808 in Münster in Graubünden, gest. 1865 in Ingenbohl), dessen Tätigkeitsfeld im Sinne eines antirationalistischen Erziehungs- und Sozialideales in umfassendem Sinne Familie, Schule, Spital und Fabrik betraf. Florentini gründete 1856 zusammen mit der Oberin des Kreuzspitales in Chur, Maria Theresia Scherer (geb. 1825 aus Meggen bei Luzern), den Orden der Barmherzigen Schwestern vom Heiligen Kreuz mit Sitz in Ingenbohl am Vierwaldstätter See «für alle Gebiete der christlichen Caritas». Es ist dies ein bis heute noch sehr aktiver Orden mit Ausdehnung über Südeuropa, Asien und die USA.

Dort in Ingenbohl lernte bei einem Erholungsaufenthalt der Dorfpfarrer von Herten, Karl Rolfus, diese Schwesternschaft kennen. Er selbst hatte eine bewegte Vergangenheit mit u. a. einer Klostergründung in der Schweiz (St. Anna in Steinenberg bei Schwyz), in das zahlreiche Mädchen aus dem Glottertal und dem Hotzenwald aufgenommen wurden. Als dort die Askese unvernünftige Formen annahm und die Kantonalverwaltung die Schwestern ausgewiesen hatte, zog er mit ihnen nach Ottmarsheim im Elsaß, wurde jedoch dort zu einer Gefängnisstrafe verurteilt, weil er ohne Genehmigung der Französischen Republik ein Kloster in Frankreich gegründet habe. Nach Herten war er mehr oder weniger strafversetzt; als er die Tätigkeit der Kreuzschwestern in Ingenbohl kennengelernt hatte, konnte er den Orden 
bewegen, in seiner Pfarrei für eine Krankenpflegestation Schwestern zur Verfügung zu stellen.

Zwei Jahre später inspizierte die Generaloberin Maria Theresia Scherer diese kleine Station, und es entstand der eingangs erwähnte Gedanke, sich der im Lande zahlreichen Schwachsinnigen anzunehmen und damit am Beispiel der Schwachsinnigenpflege ein Exempel christlicher Barmherzigkeit zu statuieren. Praktisch hieß dies, den Behinderten ein menschenwürdiges Dasein zu verschaffen und sie vor allem so weit als möglich religiös auszubilden, um sie am kirchlichen Leben teilnehmen zu lassen; eine ausgesprochene Heil- oder Unterrichtsanstalt war zunächst nicht beabsichtigt, wie die Prospekte zur Anstaltsgründung ausweisen.

Bereits acht Jahre nach der Gründung 1879 wurden in Herten 210 Behinderte von 70 Mitarbeitern betreut; das St. Josefshaus wurde zur fünftgrößten Behinderteneinrichtung Deutschlands. Zu Beginn des Jahrhunderts waren es bereits 400 Pfleglinge; für die Epileptiker wurde ein eigenes Haus (Laurentiushaus) gebaut.

Der nach außen hin lange dominierende Charakter einer Bewahranstalt wurde im übrigen in der Zeit des Nationalsozialismus möglicherweise zu einem Positivum, indem Erzbischof Conrad Gröber in seinen mutigen Eingaben an das Reichsinnenministerium von der «großen katholischen Fürsorgeanstalt für Halb- und Ganzidioten Herten»sprach, deren Bewahr-

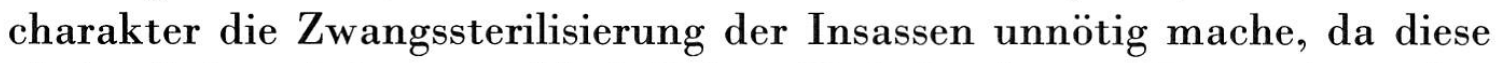
«keine Gelegenheit zu geschlechtlichem Verkehr» hätten. Dennoch wurden auch aus Herten 345 Behinderte, wie es hieß, "planwirtschaftlich verlegt» und in Zwiefalten, Grafeneck und Hadamar getötet. Davon konnten - dies ist charakteristisch für die vielen Dimensionen dieser grausamen politischen und menschlichen Tragödie - 126 gerettet werden, teils durch Übernahme ins Angestelltenverhältnis, teils durch den mutigen Einsatz von Mitarbeitern, Ordensfrauen und Angehörigen. Es gab dort den Vater, der nach Hadamar fuhr und seinen behinderten Sohn praktisch aus der Todeskammer holte, aber auch die Mutter, die ihren behinderten Sohn freiwillig der SS übergab, damit er mindestens dieses Opfer für den «Führer» bringen konnte.

Karl Rolfus, der das St. Josefshaus mit 60 Jahren gegründet hat, konnte als Leiter des Heimes auch noch das 25jährige Jubiläum mitfeiern und gab die Leitung erst 1906, 87jährig, ab. Er starb 1907 am 2. März mit 88 Jahren; im St. Josefshaus wurden zu diesem Zeitpunkt 550 geistig Behinderte betreut. Heute sind es 650 geistig Behinderte, die in 62 Wohngruppen untergebracht sind, mit 520 Mitarbeitern, einem ärztlichen Dienst, Sonder- 
schulen, Versorgungsbetrieben und beschützenden Werkstätten - übrigens weiterhin geleitet von einem Geistlichen, der allerdings zu den führenden Heilpädagogen der Bundesrepublik zählt, und immer noch betreut von den Ingenbohler Schwestern aus der Schweiz.

Es ist jedoch unübersehbar - und dies ist ein wichtiger historischer Aspekt des Problems -, daß es sich im Prinzip bei Anstalten wie Herten um Ghettos der Barmherzigkeit handelt - eine inzwischen rundherum vorbildlich, sachlich, wissenschaftlich und praktisch hervorragend funktionierende Institution, die jedoch nur in der engeren Umgebung bei der Bevölkerung bekannt ist. Der geistig Behinderte kommt im medizinischen Curriculum nicht vor; der Ortsname Herten ist im benachbarten Freiburg oder Basel im wesentlichen unbekannt.

Im Ursachenkatalog des sozialen Vorurteiles stehen die historisch bedingten Gründe an erster Stelle, gefolgt von den einseitigen Informationen, den Minderwertigkeits- und den Unsicherheitsgefühlen. Gerade im Falle der Schwerbehinderung stehen wir eingewurzelten Ängsten, Vorurteilen und traditionsmäßig geübten diskriminierenden Verhaltensweisen gegenüber, die ihr Gewicht immer noch vorwiegend aus weiterwirkenden Elementen der Tradition beziehen. 


\section{Literatur}

Damerow, Heinrich: Zur Cretinen- und Idiotenfrage. Allg. Zschr. für Psychiatrie und psychisch-gerichtl. Medizin, Bd.15, Berlin 1858, S.499-545.

Feja, Marianne: Das Krüppelkind. Ein Beitrag zur Geschichte der Körperbehindertenfürsorge. Med. Diss. Freiburg 1975.

Gadient, V.: $\quad$ Der Caritasapostel Theodosius Florentini, 2.Aufl., Luzern 1946.

Hänsel, Dagmar: Die «physiologische Erziehung» der Schwachsinnigen (Edouard Séguin 1812-1880). Freiburger Forschungen zur Medizingeschichte, N.F. Band 3, Freiburg 1974.

Kuhlo, Barbro: $\quad$ Der kindliche Schwachsinn als diagnostisches, therapeutisches Problem im 19. Jahrhundert. Medizinhistorisches Journal 9 (1974), 125-151.

Martine, E.: $\quad$ Mutter Maria Theresia Scherer, Ingenbohl 1959.

Mauri, Heinrich: $\quad$ Die Entwicklung des Kretinenproblems, Med. Diss. Freiburg 1976.

Nettlau, Manfred: $\quad$ Das St. Josephshaus in Herten, Med. Diss. Freiburg 1981.

Sagi, Alexander: $\quad$ Behinderte. In: Wörterbuch medizinischer Grundbegriffe. Hrsg.: E. Seidler. Freiburg 1979, S. 55-58.

Seidler, Eduard: Die Entwicklung des Rehabilitationsverständnisses. Krankenpflege 10 (1980), 338-341.

Steinacher, E.: $\quad$ Die Ingenbohler Schwestern, Ingenbohl 1960.

Strasilla, Annette: Die Heilpädagogik in der ersten Hälfte des 19. Jahrhunderts unter besonderer Berücksichtigung der Bestrebungen in der Anstalt Levana (1856-1865) in Wien, Med. Diss. Freiburg 1974.

\section{Summary}

Herten is a village near Switzerland, but pertaining to Baden. Karl Rolfus, the pastor of Herten, was once staying at the Lake of the Four Forest Cantons. Here he became acquainted with the work of the order of the Sisters of Mercy, founded and directed by Maria Theresia Scherer at Ingenbohl. Rolfus established a hospital care home at Herten, and some sisters were ready to come. Maria Theresia Scherer visited it and proposed to do something for the cretins and mental defective persons. Rolfus was sixty years old, when he opened this detention home, in 1879. He was in charge of it till 1906. The home still exists, and sisters from Ingenbohl are helping to take care of 650 feeble minded inhabitants.

Prof. Dr. med. Eduard Seidler

Institut für Geschichte der Medizin

der Albert-Ludwigs-Universität

Stefan-Meier-Straße 28

D-78 Freiburg i.Br. 